\title{
Patterson-Kelly Syndrome: A Single Centre Experience
}

\author{
Ramesh Satarkar \\ Satarkar Gastroenterology Centre, Aurangabad, Maharashtra, India
}

Email address:

rpsatarkar@rediffmail.com

\section{To cite this article:}

Ramesh Satarkar. Patterson-Kelly Syndrome: A Single Centre Experience. International Journal of Gastroenterology. Vol. 4, No. 2, 2020, pp. 41-44. doi: 10.11648/j.ijg.20200402.13

Received: June 28, 2020; Accepted: July 16, 2020; Published: August 4, 2020

\begin{abstract}
Background: Patterson Kelly syndrome is a rare entity, presenting with a triad of Iron deficiency anemia, Dysphagia and Esophageal webs. The syndrome is extremely rare and its incidence is decreasing probably due to improvements in nutritional standards. The syndrome is enigmatic in the sense that not all patients may be having anemia, the syndrome is associated with various autoimmune conditions, webs are located in post-cricoid region only and there is increased prevalence of upper gastrointestinal malignancies in these patients. The symptoms respond to iron replacement but many patients require rupture of web for relief of dysphagia. Savary dilators, TTS balloon, electro-cautery have been variably used to break the web. Objectives: 1. To study the profile of Patterson Kelly syndrome at our center; 2 . To study if the endoscope itself can be used as effective dilator for rupturing the web; 3 . To study the effect of iron replacement in these patients; 4. To study the occurrence of upper gastrointestinal malignancies in this condition. Material and methods: We report our experience of sixteen patients with Patterson Kelly syndrome over last fifteen years. This is a retrospective, single center, observational study. All patients had demonstrable web on endoscopy. The endoscope itself was used as a dilator to break the web in more than half of the patients. The endoscope being rigid instrument can easily break the thin web during diagnostic endoscopy itself. If the opening was too narrow, TTS balloon or Savary Guilard dilators were used. One patient had a perforation while using endoscope as the dilator. Five patients required more than one sitting of dilatation for achieving relief of dysphagia or for recurrent web formation. Most patients received intravenous iron supplementation. Ten patients had complete relief of dysphagia without recurrence. One patient had concomitant esophageal squamous cell carcinoma in lower esophagus which became evident when the scope was passed distally, post dilatation of the web. Conclusions: Although rare, Patterson Kelly syndrome continues to exist and can be effectively treated by iron replacement, dilatation and rupture of esophageal webs using various methods. The web can be ruptured by careful maneuvering of the endoscope itself. Intravenous iron replacement can be used prior to the procedure which can ameliorate the symptoms and reduce the need for the dilatation or can make it easier. Patients need surveillance for upper gastrointestinal malignancies however exact guidelines in this regard are lacking.
\end{abstract}

Keywords: Patterson Kelly Syndrome, Esophageal Webs, Dysphagia, Iron Deficiency Anemia

\section{Introduction}

Patterson-Kelly syndrome (in UK), also known as Plummer Vinson Syndrome (in USA), or Sideropenic dysphagia (due to presence of iron deficiency) is a rare syndrome, characterized by dysphagia, iron deficiency and presence of post-cricoid esophageal web. Patterson and Kelly first described clearly the entire clinical picture and the components of the syndrome $[1,2]$. Rarely patients may not have iron deficiency anemia. Most of the published literature is in the form of case reports barring exception of a few case series with number of patients exceeding two digits [3-6]. We report 16 cases in last 15 years at our center.

The patients usually have long standing dysphagia, often have multiple nutritional deficiencies which could be one of the factor leading to mucositis and web formation. Other disorders reported to be associated with this syndrome include Celiac disease, Crohn's disease, Rheumatoid arthritis and thyroid disease raising the possibility immune dysregulation may be involved in its pathogenesis although this remains to be proven $[7,8]$.

The web is typically eccentric, located in post-cricoid esophagus can get ruptured unintentionally during diagnostic 
endoscopy if it is very thin or can be ruptured using variety of dilatation techniques using Savary Guilard dilators, TTS balloons, or electro-cautery $[4,9,10]$. For the first time, we report a purposeful use of the endoscope for rupturing the web. In half of our patients no other dilator was used, the procedure could be completed in single sitting, under conscious sedation with good results. There is increased risk of esophageal and other gastrointestinal malignancies in these patients. One of our patient had a concomitant lower esophageal squamous cell carcinoma.

\section{Material and Methods}

This is a retrospective, observational, single center study. Case records of all patients presenting with dysphagia and esophageal web were searched from 2005 to 2020. Patient demography, available investigations including complete blood count, serum iron studies, barium swallow study results, endoscopy findings, type of dilatation method, results of dilatation, follow up visits for recurrence, complications if any were looked for and analyzed. Anemia was diagnosed when Hemoglobin was less than 11 gm.\%, sideropenia when serum iron and ferritin were below normal or iron deficiency indicated by microcytic hypochromic anemia on red cell indices or on peripheral smear examination. Barium swallow was done in patients in whom the post cricoid stenosis was so tight that web could not be seen properly during diagnostic endoscopy. It provided not only the confirmation of diagnosis but also the road map for dilatation.

Being a retrospective study, done in a private health care set up in India, where patients have to pay for all the investigations themselves and as most do not have insurance, all the investigations could not be done in every patient.

\section{Results}

Sixteen patients were identified to have esophageal webs on endoscopy during the study period.

All patients were women, in age group 35 to 65 years.

15 had dysphagia but one did not. Duration of dysphagia ranged from 15 days to 216 months. 8 patients had dysphagia more than one year. One patient had odynophagia, 4 had glossitis, and 11 were subnormal in weight for height.

Hemoglobin ranged from 7 to $13 \mathrm{gm}$. with an average of $9.48 \mathrm{gm}$. Eleven patients had anemia $(\mathrm{Hb}<11 \mathrm{gm} . \%)$, four had normal hemoglobin and data was not available for one patient. Eleven patients had subnormal serum Ferritin and 9 had subnormal serum iron levels. Of the four patients with normal hemoglobin two had sideropenia, one had normal serum iron studies and available data was incomplete in one patient

Barium swallow was done in seven patients in whom scope could not be negotiated into esophagus during diagnostic endoscopy. It showed classical post-cricoid web in all (Figure 1).

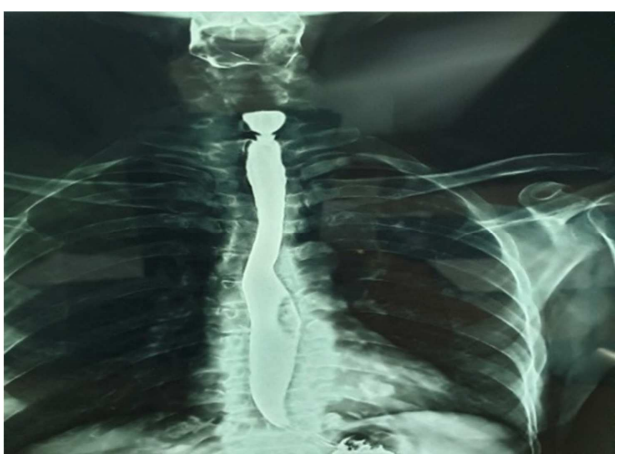

Figure 1. Barium swallow showing post cricoid web.

All patients had a demonstrable web on upper gastrointestinal endoscopy (Figure 2).

All procedures were done under local anesthesia and conscious sedation. In 7 patients the web was broken during diagnostic endoscopy using the scope as the dilator. In 3 patients 14 or $15 \mathrm{~mm}$ TTS balloon alone was used. In one patient each Savary - Guilard dilators, Savary dilators plus TTS balloon, TTS balloon plus endoscope were used as dilators. One patient required needle knife to cut the web. Total 22 sessions were required in 14 patients to achieve relief of dysphagia. Nine required single session, three required two sessions and one each required 3 and 4 sessions for complete relief of dysphagia (Figure 3).

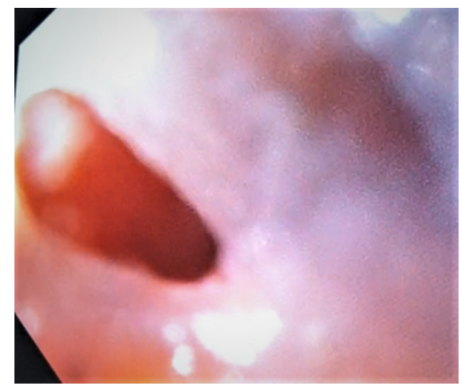

Figure 2. Endoscopic picture of circumferential web.

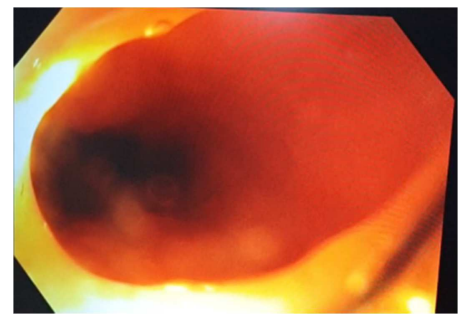

Figure 3. Post dilatation.

There was one perforation in post-cricoid region with use of endoscope as the dilator. It was managed conservatively.

Iron replacement was done with intravenous iron in 7 patients, with oral route in 5 patients, by both oral and intravenous route in 2 patients. Two patients, one who had normal serum iron studies and one in whom data was incomplete, did not receive any supplement.

Average follow up was 24 month with a median of six months. Ten patients had complete relief of dysphagia, three were lost to follow up, one patient refused dilatation 
procedure, one is being scheduled for dilatation procedure and one with squamous cell carcinoma of distal esophagus is being treated for the same. The patient who refused the procedure and on who is being scheduled for the procedure had significant improvement in dysphagia after intravenous iron replacement.

One patient had squamous cell carcinoma in lower esophagus which became evident when scope was passed in distal esophagus, after dilation of the post cricoid web.

\section{Discussion}

Patterson-Kelly or Plummer Vinson syndrome is a rare entity which continues to be enigmatic despite its first description more than a century ago $[1,2]$. Most of the recent published literature is in the form of case reports with few case series [3-6]. This speaks of its rarity and also reduction in the incidence probably due to improvement in nutritional standards. It was more common in Scottish women earlier [11]. Now most reports come from Asia. In a study from Peshawar this syndrome accounted for $5 \%$ of cases of dysphagia [12]. The syndrome is more common in women, with women to men ratio as high as 8.5:1 Rare case reports of the syndrome in men also appear in literature $[13,14]$. In our series all patients were women. The syndrome is common in middle aged women and symptoms are long standing. Similar results were found in our study.

Exact cause for web formation, role of iron deficiency and localization of the web to post-cricoid region remains unknown. Iron deficiency anemia is more prevalent in patients of esophageal webs; its treatment has been found to lead to improvement in dysphagia and disappearance of webs [15-17]. One of the hypotheses put forwards is that deficiency of iron dependent oxidative enzymes leads to myaesthenic changes in muscles, atrophy of mucosa and eventual formation of webs. However only $10 \%$ patients of IDA develop webs implying other factors are also important. Autoimmunity with autoantibodies against a yet unidentified antigen has been another hypothesis. The syndrome is associated with thyroiditis, rheumatoid arthritis, celiac disease, Pernicious anemia [7, 8]. However association and causality are different. Probably all these conditions have iron deficiency state which could be the common link between the two conditions.

Heterotopic gastric mucosa with inlet patch which gets injured and heals by fibrosis leading to mucosal web formation is one more hypotheses. However in our unpublished experience inlet patches are more common and are generally asymptomatic.

The location of the webs to post-cricoid region is explained by the trauma theory which proposes that the postcricoid area being bound by skeletal muscles takes maximum trauma during solid bolus swallowing. The trauma coupled with various deficiencies leads to low grade inflammation leading to healing by fibrosis and formation of webs [18].

Thus the syndrome appears multifactorial in origin and exact contribution of each factor is variable and different in different subset of patients.

Dysphagia is seen when luminal diameter is less than 12 $\mathrm{mm}$. Usually it is long standing and being mechanical, more for solids. Iron deficiency anemia usually manifests with easy fatigue, breathlessness, pallor, koilonychia. Other nutritional deficiencies can give rise to glossitis, angular cheilitis. In our series majority patients were sub-normal in weight, four had stomatitis and glossitis.

Microcytic hypochromic or dimorphic anemia and reduced serum ferritin are seen. The cause of anemia especially blood loss if suspected, needs further investigations.

Barium swallow especially cine-fluoroscopy of upper third is required to demonstrate the web. Large bolus, thicker barium and lateral views in standing position increase the yield of capturing the web on imaging. Posterior webs are more common than anterior or circumferential ones. The observation of jet phenomenon, indicative of forceful passage of barium through a narrow opening, is seen more commonly in symptomatic patients [19].

Video endoscopy is of diagnostic as well as of therapeutic value. Resistance to passage of scope in post-cricoid area should arouse the suspicion. Sedation is preferred for exact assessment. At endoscopy webs appear eccentric, thin, at times circumferential, producing fish mouth appearance. Web may need to be broken or dilated for distal viewing.

Correction of anemia is the first step and can lead to disappearance of webs and resolution of dysphagia. We prefer intravenous iron therapy as it is a rapidly acting method. Other nutritional deficiencies if any also need to be corrected.

The webs can be dilated using Savary-Guilard dilators [9] or TTS Balloon [4]. Choice depends on available dilators and infrastructure facilities. Savary-Guilard dilators need access to fluoroscopy. TTS dilators may not be useful for very elastic, stretchable webs. Dysphagia relief requires rupture of web. Many a times maneuvering the scope and controlled force can rupture thin web, facilitating passage of scope. However this needs to be done carefully and with judgment. Untoward use of force can cause perforation. Sometimes IT knife or papillotome wire can be used to cut circumferential web [10]. Four quadrant controlled mucosal cuts are enough for relief of symptoms. Some patients may require more than one sitting. We used the passage of scope with careful maneuvering as the sole method for web rupture in seven patients. We had one perforation which was managed conservatively. We prefer TTs balloon over Savary-Guilard dilators as it is easy to use, dilatation can be done in same sitting as diagnostic endoscopy and does not require fluoroscopy. Some patients require more than one sitting. In our series 5 patients required multiple sittings.

Prognosis is generally excellent. Patients need surveillance for post-cricoid and other gastrointestinal malignancies as this syndrome is considered to be a pre- malignant state [7, 18]. One of our patients had concomitant squamous cell carcinoma in mid-esophagus which became evident when scope was passed distally post-dilatation. 


\section{Conclusions}

Patterson Kelly syndrome is a rare but real entity. Its exact etio-pathogenesis is not clear but appears multifactorial.

The syndrome occurs predominantly in women. Patients generally have long standing dysphagia. Sideropenia is almost always there even if the hemoglobin is normal. If the esophageal lumen is adequate $(>12 \mathrm{~mm})$, just iron replacement may be enough, but if there is significant dysphagia, some form of dilatation technique will be required for relief of dysphagia. The endoscope itself can be used to rupture the web by careful maneuvering. Some patients require TTS balloon or Savary-Guilard dilators or electrocautery for rupture of the web.

These patients need surveillance for gastrointestinal malignancies as this is considered to be a premalignant condition.

\section{References}

[1] Paterson DR. A clinical type of dysphagia. Proceedings of the Royal Society of Medicine. 1919 May; 12 (Laryngol_Sect): 235-9.

[2] Brown-Kelly A. Spasm at the entrance of the oesophagus. J Laryngol Otol. 1919 Aug; 34: 285-9.

[3] Bakari G, Benelbarhdadi I, Bahije L, El feydi Essaid A. Endoscopic treatment of 135 cases of Plummer-Vinson web: a pilot experience. Gastrointestinal endoscopy. 2014 Oct 1; 80 (4): 738-41

[4] Goel A, Lakshmi CP, Bakshi SS, Soni N, Koshy S. Singlecenter prospective study of Plummer-Vinson syndrome. Diseases of the Esophagus. 2016 Oct 1; 29 (7): 837-41.

[5] Chhabra P, Khurana H. Image Diagnosis: Plummer-Vinson Syndrome: An Unusual Cause of Dysphagia. Perm J. 2018; 22: 18-035. doi: 10.7812/TPP/18-035. PMID: 30005727; PMCID: PMC6045507.

[6] Changela, K., Haeri, N. S., Krishnaiah, M. et al. PlummerVinson Syndrome with Proximal Esophageal Web. $J$ Gastrointest Surg 20, 1074-1075 (2016). https://doi.org/10.1007/s11605-015-3051-5.

[7] Chisholm M. The association between webs, iron and postcricoid carcinoma. Postgraduate Medical Journal. 1974 Apr 1; 50 (582): 215-9.

[8] Makharia GK, Nandi B, Garg PK, Tandon RK. Plummer
Vinson syndrome: unusual features. Indian journal of gastroenterology: official journal of the Indian Society of Gastroenterology. 2002; 21 (2): 74.

[9] Salihoun M, Kabbaj N, Raissouni F, Chaoui Z, Mohamed A, Naima A. Safety and effectiveness of endoscopic SavaryGillaard Bougies dilation in Moroccan Plummer-Vinson syndrome patients. International Scholarly Research Notices. 2013; 2013.

[10] Ohtaka M, Kobayashi S, Yoshida T, Yamaguchi T, Uetake T, SatoT, Hayashi A, Kanai M, Yamamoto T, Hatsushika K, Masuyama K. Use of Sato's curved laryngoscope and an insulated-tip knife for endoscopic incisional therapy of esophageal web. Digestive Endoscopy. 2015 May; 27 (4): 522-6.

[11] Elwood PC, Pitman RG, Jacobs A, Entwistle CC. Epidemiology of the Paterson-Kelly syndrome. The Lancet. 1964 Oct 3; 284 (7362): 716-20.

[12] Hussin A, Din IU, Arif A, Shah SM, Hafeez M. Plummer Vinson Syndrome in Patients Presenting With Dysphagia. Journal of Medical Sciences. 2017 Mar 6; 25 (1): 24-6.

[13] Swain SK, Panigrahy R, Sahu MC. Plummer Vinson syndrome in a male and his chromosomal study-A case report. Egyptian Journal of Medical Human Genetics. 2015; 16 (3): 283-6.

[14] Priyadarshini Karthikeyan, Nalini Aswath, Ramesh Kumaresan Plummer Vinson Syndrome: A Rare Syndrome in Male with Review of the Literature. Case Reports in Dentistry; Volume 2017 | Article ID 6205925|5 pages |https://doi.org/10.1155/2017/6205925.

[15] Bredenkamp JK, Castro DJ, Mickel RA. Importance of iron repletion in the management of Plummer-Vinson syndrome. Annals of Otology, Rhinology \& Laryngology. 1990 Jan; 99 (1): $51-4$.

[16] Dantas RO, Villanova MG. Esophageal motility impairment in Plummer-Vinson syndrome. Digestive diseases and sciences. 1993 May 1; 38 (5): 968-71.

[17] Dantas RO. Iron deficiency and dysphagia. American Journal of Gastroenterology. 1999 Oct 1; 94 (10): 3072-3.

[18] Jones RM. The Paterson-Brown Kelly syndrome: its relationship to iron deficiency and postcricoid carcinoma. The Journal of Laryngology \& Otology. 1961 Jun; 75 (6): 544-61.

[19] Waldenstrom J, Kjellberg SR. The roentgenological diagnosis of sideropenic dysphagia. Acta radiologica. 1939 Jan 1; 20 (6): 618-38. 